\title{
Factores asociados a violencia horizontal intrahospitalaria en el personal de enfermería: revisión sistemática
}

\section{Factors associated to intra-hospital horizontal violence against nursing personnel: a systematic review}

\section{Fatores associados a violência horizontal intrahospitalar no pessoal de enfermagem: revisão sistemática}

\author{
I. Llanos-Jiménez ${ }^{\text {* }}$, E. Rivas-Riveros ${ }^{\mathrm{b}}$, M. López-Espinoza $^{\mathrm{c}}$ \\ ${ }^{a}$ Facultad de Enfermería, Universidad de Concepción, Chillán- Chile

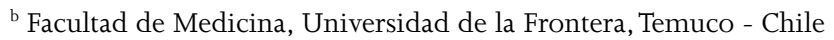 \\ ${ }^{\mathrm{c}}$ Escuela de Nutrición y Dietética, Facultad de Salud, Universidad Santo Tomás, Sede Talca - Chile \\ Recibido: 7 agosto 2017 \\ Aceptado: 22 mayo 2018
}

\section{Resumen}

Objetivo: Determinar factores de riesgo asociados a violencia horizontal intrahospitalaria en el personal de enfermería.

Metodología: Revisión sistemática sobre estudios epidemiológicos que analizaron violencia horizontal y sus factores asociados. Se consultó Pubmed, ProQuest, ScienceDirect, Ebsco-Host, CINHAL, ISI Web of Science y Biblioteca Virtual en Salud. Se aplicó lectura crítica (STROBE), se otorgó nivel de evidencia y grado de recomendación con AHQR.

Resultados: De 629 registros, 7 artículos fueron incluidos en la revisión sistemática. Todos obtuvieron nivel de evidencia III y grado de recomendación B. La prevalencia de violencia horizontal osciló entre 21.179.4\%. Factores asociados a violencia horizontal fueron: lugar de trabajo, satisfacción con el trabajo, relaciones entre pares, plan para abandonar el trabajo y presencia de políticas relativas al acoso laboral. Conclusiones: Existe escasa evidencia del tema y de calidad moderada. Se hace necesario reconocer prevalencia de violencia horizontal y sus factores asociados, para diseñar estrategias de prevención en cultura laboral.

Palabras clave: Violencia laboral; violencia horizontal; lugar de trabajo; salud ocupacional; enfermería del trabajo; Chile. 


\section{Abstract}

Objective: To determine risk factors associated to intra-hospital horizontal violence against nursing personnel.

Methodology: This is a systematic review about epidemiological studies analyzing horizontal violence against nurses and its associated factors. Pubmed, ProQuest, ScienceDirect, Ebsco-Host, CINHAL, ISI Web of Science and Biblioteca Virtual en Salud databases were consulted. A critical lecture strategy (STROBE) was followed. AHQR-based Level of Evidence and Recommendation Degree were assigned.

Results: From 629 registers, 7 articles were included in the systematic review. All received a Level of Evidence III and a Degree of Recommendation B. The prevalence of horizontal violence was found to be in the range of $21.1 \%$ to $79.4 \%$. Associated risk factors were: place of work, job satisfaction, relationship with colleagues, intention to leave, and work harassment related policies.

Conclusions: There is scant and limited in quality evidence on the topic. It becomes necessary to acknowledge the prevalence of intra-hospital horizontal violence against nurses and its associated factors in order to design prevention strategies which can strengthen the labor culture.

Keywords: Workplace Violence; horizontal violence; place of work; occupational health; occupational health nursing; Chile.

\section{Resumo}

Objetivo: Determinar fatores de risco associados a violência horizontal intrahospitalar no pessoal de enfermagem.

Metodologia: Revisão sistemática sobre estudos epidemiológicos que analisaram violência horizontal e seus fatores associados. Consultou-se Pubmed, ProQuest, ScienceDirect, Ebsco-Host, CINHAL, ISI Web of Science e Biblioteca Virtual en Salud. Aplicou-se leitura crítica (STROBE), outorgou-se nível de evidência e grau de recomendação com AHQR.

Resultados: De 629 registros, 7 artigos foram incluídos na revisão sistemática. Todos obtiveram nível de evidência III e grau de recomendação B. A prevalência de violência horizontal oscilou entre 21.1-79.4\%. Fatores associados a violência horizontal foram: lugar de trabalho, satisfação com o trabalho, relações entre pares, plano para abandonar o trabalho e presência de políticas relativas ao assédio laboral.

Conclusões: Existe escassa evidência do tema e de qualidade moderada. É necessário reconhecer prevalência de violência horizontal e seus fatores associados, para desenhar estratégias de prevenção em cultura laboral.

Palavras chave: Violência laboral; violência horizontal; lugar de trabalho; saúde ocupacional; enfermagem do trabalho; Chile.

\section{Introducción}

La violencia horizontal es frecuente en enfermería, ampliamente documentada y reportada ${ }^{1,2}$, debido a ello se ha considerado a enfermería la principal ocupación en riesgo ${ }^{3}$, sin embargo, la situación pocas veces se denuncia por temor a represalias ${ }^{4}$. Este término ha sido definido y traducido de varias maneras ${ }^{5}$, lo cual hace complejo su estudio, es por ello que en el año 2010 se creó el acrónimo BHHV (bullying, harassment (acoso) and horizontal violence (violencia horizontal), para describirlo como un concepto multifactorial asociado a comportamientos repetitivos, ofensivos, abusivos, intimidantes o insultantes, abuso de poder o sanciones injustas que hacen a los receptores sentirse humillados, vulnerables o amenazados, los cuales crean estrés y disminuye la confianza en sí mismos ${ }^{6}$.

La violencia horizontal fue explicada en primer lugar por la Teoría de la Opresión, desarrollada por el pedagogo brasileño Paulo Freire ${ }^{7}$, la cual fue retomada por Susan Roberts (1983) para aplicarla a Enfermería ${ }^{8}$. Señala que, en sus frustraciones por la falta de poder y autonomía, actúan con violencia hacia los otros ${ }^{9}$. Esta conducta basada en interacciones antagónicas, deshonrosas y descortés entre el personal de enfermería que trabaja en niveles de organización comparables, son caracterizadas por malentendidos 
divisivos y luchas internas ${ }^{10}$, que acaban por perjudicar, despreciar y devaluar el valor de persona al negar sus derechos humanos básicos, a través de un comportamiento verbal (gritos, críticas persistentes, menospreciar) o no verbal (ignorar, suspirar, juego de ojos), actos físicos como empujar o lanzar elementos alrededor de la víctima ${ }^{11}$, socavar y sabotear (retener información, negarse a ayudar) o mantener confianzas rotas (chismes, rumores) ${ }^{12}$. Tal intimidación entre colegas del mismo nivel jerárquico es el resultado de una baja autoestima personal y una mala identidad grupal ${ }^{13}$.

Un estudio concluyó que el 70\% del personal de enfermería había vivido una experiencia de violencia, y son los perpetradores (55\%), los propios colegas de distintos estamentos ${ }^{14}$. Otro estudio reporta que el $54.8 \%$ del total de menciones de algún tipo de violencia en contra de egresados de la licenciatura en enfermería fue ejercida por un colega ${ }^{15}$. El problema más serio de violencia hacia el personal, corresponde cuando es horizontal, es más difícil de olvidar y causa episodios mucho más angustiantes, en comparación a conductas similares efectuadas por médicos o pacientes ${ }^{16}$.

Es un fenómeno muy común que tiene consecuencias perjudiciales para el personal de enfermería, los pacientes, las instituciones de salud, y para la profesión de enfermería en si $^{17}$, su presencia aumenta la intención de dejar la profesión ${ }^{18}$. Además, la violencia horizontal implica, para quien la recibe una baja autoestima, depresión, hostilidad, resultados negativos con los pacientes, sentimientos de impotencia; también puede vincularse al nivel administrativo como la incapacidad para reclutar personal, el aumento de la rotación, relaciones dañadas, falta de retención y cooperación ${ }^{19}$, 20 , la disminución del tiempo de trabajo, disminución de la productividad y el aumento de los costos médicos, ya que aumentan los errores y eventos adversos ${ }^{21}$.

En general es responsabilidad de enfermería diseñar, implementar y evaluar programas que respondan a las necesidades para mejorar la calidad de vida durante el ciclo vital ${ }^{22}$, más si el objeto del estudio es la misma profesión. Gestionar e implementar planes de promoción para la salud es parte de la profesión, con fuerte énfasis en la participación de los mismos trabajadores ${ }^{23}$. En particular ante esta problemática, implica entregar herramientas suficientes para empoderar al personal y con ello evitar que sucedan eventos de violencia laboral ${ }^{24}$. Existe escaso reconocimiento de la violencia lateral en el lugar de trabajo (también denominada violencia horizontal), el conocimiento del concepto y los métodos para evitar su incidencia, por lo cual se necesitan métodos de intervención educativa eficientes ${ }^{25}$ y antes de ello, se deben identificar los principales factores ${ }^{26}$ que se asocian a este problema.

Por lo tanto, el objetivo de este trabajo fue determinar factores que se asocian a violencia horizontal en profesionales de enfermería que laboran en el sector intrahospitalario, por medio de una revisión sistemática y posterior metaanálisis de los datos recolectados.

\section{Materiales y Métodos}

El diseño fue Revisión sistemática (RS). Los estudios elegibles reclutaron a profesionales de enfermería que laboran en el sector intrahospitalario.

Fueron criterios de inclusión, investigaciones epidemiológicas de caso-control, transversales y de cohorte. Se excluyeron estudios donde no se apreciaba con claridad el método de medición de esta variable, así como trabajos donde se analizaban episodios violentos o agresión de pacientes y/o familiares hacia el personal de enfermería; acoso laboral en otras ramas profesionales; estudios teóricos, estudios que abordaban el efecto de la violencia y opiniones de expertos.

Los trabajos incluidos fueron: estudios que hayan considerado a trabajadores remunerados y dependan de una jefatura directa. Fueron excluidos estudios con auxiliares de enfermería, estudiantes de enfermería, profesionales que laboraban en más de un hospital y quienes ejercían docencia dentro del recinto.

El trabajo de búsqueda y selección de estudios se realizó entre abril y septiembre del año 2016.

En la estrategia de búsqueda y recolección de datos, dos revisores independientes y un tercero con formación en búsqueda bibliográfica, identificaron en el título y resumen los artículos que contenían información del tema en estudio. De los artículos donde existieron dudas, se leyó la metodología para 
tomar la decisión. Posteriormente, los revisores clasificaron los artículos como "incluido”, "excluido” y "dudoso".

Para la localización y selección de estudios. Se recurrió a las bases de datos electrónicas Pubmed, ProQuest, ScienceDirect, Ebsco-Host, CINHAL, Web of Science y Biblioteca Virtual en Salud. Se utilizaron descriptores en español e inglés según la base de datos, se aplicaron las equivalencias del lenguaje estructurado MeSH (Medical Subjects Headings). También, se utilizaron palabras no controladas extraídas de las palabras clave de los artículos seleccionados. La combinación de las palabras claves fue: (((horizontal violence) OR (lateral violence) OR (oppression) OR (Workplace psychological violence) OR (harassment aggression abuse incivility) OR (horizontal mobbing) OR (horizontal bullying) OR (negative behaviour among peers) OR (“WorkplaceViolence”[Mesh])) AND (colleagues OR intra nurse OR nurses OR nursing) AND (risk factors OR associated OR association)).

Luego, dos revisores enmascarados examinaron cada artículo seleccionado y fueron elegidos para lectura crítica con pautas de evaluación de estudios observacionales de la colaboración Strengthening the Reporting of Observational Studies in Epidemiology (Strobe) ${ }^{27}$. Cada lector calificó de forma independiente con tres opciones perceptuales: $\mathrm{A}=$ bueno (cumple todos los aspectos de estructura y contenido que la guía Strobe solicita), B=promedio (no cumple todos los aspectos de estructura y contenido de la guía Strobe, pero no compromete de forma importante la calidad de los resultados), C=pobre (no cumple todos los aspectos de estructura y contenido de la guía, y compromete de forma importante la calidad de los resultados) y $\mathrm{D}=$ no medible, no encontrado.

Todos los estudios que se incluyeron fueron clasificados según el nivel de evidencia que aportaron, de acuerdo con la propuesta de AHQR ${ }^{28}$ (del inglés: Agency for Healthcare Rersearch and Quality).

\section{Resultados}

La búsqueda consideró 629 artículos no duplicados extraídos de seis bases de datos, no se encontró ningún resultado en la Biblioteca Virtual de Salud (BVS Salud). La Figura 1 muestra el proceso de selección de los artículos incluidos en esta RS. Los artículos que conforman la muestra concentran la fecha de publicación entre los años 2013 y 2016.

De los 7 estudios seleccionados ${ }^{5,29-34}$, todos presentaron un diseño transversal y sin grupos de comparación, donde se evaluaron modelos de regresión lineal, que incluyen coeficientes de correlación de Pearson. Provienen de Estados Unidos (4 artículos), Corea del Sur (2 artículos) y Canadá (1 artículo).

La Tabla 1 indica que la evidencia aportada por estos estudios es moderada (B), puesto que provienen de estudios observacionales con aporte metodológico y de contenido suficiente (tipo III).

Tabla 1. Evaluación cualitativa de la calidad de la evidencia

\begin{tabular}{|c|c|c|c|c|c|c|}
\hline \multirow[b]{2}{*}{ Estudio } & \multicolumn{5}{|c|}{ Calificación cualitativa por consenso* } & \multirow[b]{2}{*}{$\begin{array}{l}\text { Nivel de evidencia } \\
\text { / Grado de } \\
\text { recomendación** }\end{array}$} \\
\hline & 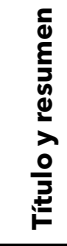 & 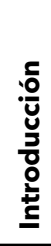 & $\begin{array}{l}\frac{n}{0} \\
\stackrel{0}{ \pm} \\
\stackrel{0}{ \pm}\end{array}$ & 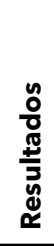 & $\frac{.00}{\underline{u}}$ & \\
\hline Budín W, Brewer C, Chao Y-Y, Covner Ch $(2013)^{5}$ & B & A & B & B & B & III / B \\
\hline Oh H, Choon D, JooY $(2016)^{29}$ & A & A & B & B & A & III / B \\
\hline Purpora C, Blegen M (2015) $)^{30}$ & A & A & B & B & B & $\mathrm{III} / \mathrm{B}$ \\
\hline Purpora C, Blegen M, Stotts N (2015) $)^{31}$ & B & A & B & B & A & $\mathrm{III} / \mathrm{B}$ \\
\hline Yun S, Kang J, Lee J-O, YiY $(2014)^{32}$ & A & A & B & B & B & $\mathrm{III} / \mathrm{B}$ \\
\hline Armmer F, Ball Ch (2015) $)^{33}$ & A & A & B & B & B & $\mathrm{III} / \mathrm{B}$ \\
\hline Blackstock S, Harlos K, Macleod M, Hardy C (2015) ${ }^{34}$ & B & A & B & B & B & $\mathrm{III} / \mathrm{B}$ \\
\hline
\end{tabular}




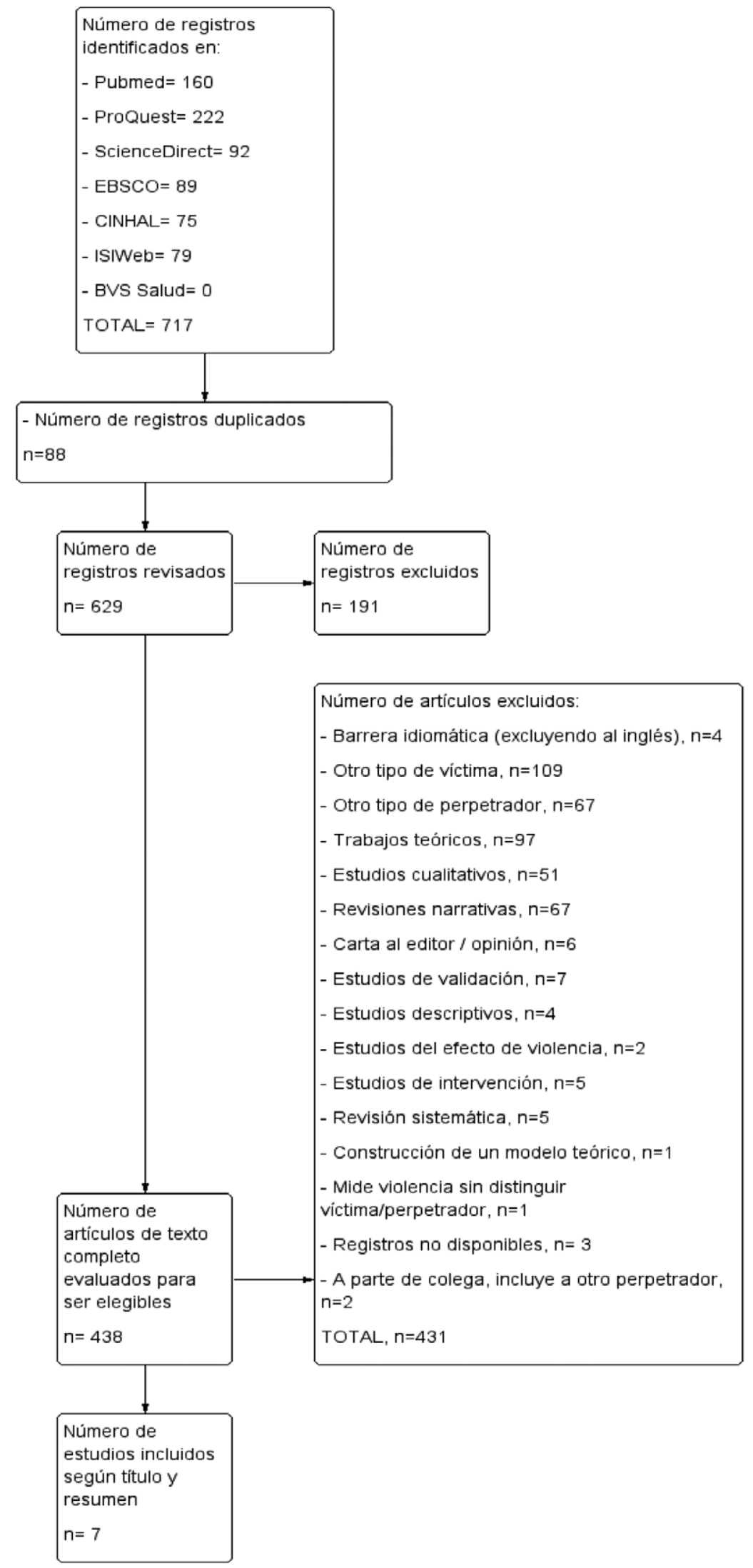

Figura I. Diagrama de flujo de los estudios seleccionados 
Respecto a la frecuencia de acoso horizontal, existe variabilidad en los estudios, por un lado, Yun, et $\mathrm{al}^{32}$, indicaron que el $22.4 \%$ del personal encuestado nunca había sido expuesto a acoso laboral y que el $10.0 \%$ de los que habían experimentado la intimidación reportaron el incidente.

Según el trabajo de Budin, et $\mathrm{al}^{5}$, el $49 \%$ del personal de enfermería experimentó abuso de sus colegas en los últimos tres meses y el 5\% percibió altos niveles de abuso, las conductas más frecuentes fueron hablar con un aire de superioridad e ignorar al colega.

Por otro lado, Purpora, et $\mathrm{al}^{31}$, indicaron que el $79.4 \%$ de los encuestados informaron haber experimentado violencia horizontal en el trabajo, al menos una vez en seis meses antes al estudio. El 58.3\% reportaron violencia horizontal mensualmente, mientras que el $21.1 \%$ manifestaron haber sido sometidos a ella semanal y/o diariamente.

Respecto al género, el principal hallazgo de los artículos analizados destaca que la mayoría de los profesionales eran de sexo femenino, sin embargo, los resultados según el género no fueron estadísticamente significativos en ninguno de los artículos, según los resultados de Yun et al ${ }^{32}$, las enfermeras obtuvieron un puntaje de violencia laboral mayor que los enfermeros (1.66 vs 1.59 ). Los resultados de Purpora, et $\mathrm{al}^{31}$ encontraron que la diferencia entre medias no fue estadísticamente significativa entre enfermeras mujeres y hombres, 1.52 versus 1.48 puntos respectivamente.

Respecto a las variables sociodemográficas, los artículos difieren en sus hallazgos respecto a la edad. Los autores Yun, et $\mathrm{al}^{32}$, indican que quienes tenían menos de 25 años presentaron un puntaje ligeramente mayor de violencia (1.77) en comparación con los mayores de 25 años (1.59), pero esta diferencia no fue estadísticamente significativa. Resultado similar a lo expuesto por Purpora, et al ${ }^{31}$. Sólo un artículo encontró una correlación entre edad y violencia horizontal, y fue el de Armmer y Ball ${ }^{33}$, que mostró que el sabotaje de enfermeras con la edad presenta una correlación directamente proporcional, aun cuando su intensidad es muy baja ( $\mathrm{r}=0.157)$.

En relación al estado civil, la investigación coreana ${ }^{32}$, indicó que el personal de enfermería soltero, tienen en mayor medida tendencia a ser víctimas de violencia, ya que obtuvieron en promedio un puntaje de violencia mayor (1.69 versus 1.48 ), respecto a los casados, esto no estadísticamente significativo. En la misma línea el estudio de Budín, et al ${ }^{5}$, observó que los solteros presentaron proporciones de moderada y alta violencia verbal de 30.1 y $40.4 \%$, respectivamente, a diferencia de los casados, quienes en un $73.7 \%$ no reportaron abuso, estos datos son estadísticamente significativos.

En el nivel educacional, el estudio coreano ${ }^{32}$ mostró que quienes eran diplomados en enfermería presentaron un puntaje de violencia mayor en comparación con las que tenían el grado académico de Bachelor y el de Master u otro superior, pero tales diferencias no fueron estadísticamente significativas. A mayor nivel educacional menor probabilidad de sufrir o ser víctima de violencia horizontal.

En relación a las variables laborales como los años de experiencia en el trabajo, los artículos difieren entre sí, mientras que Armmer y Ball ${ }^{33}$ demostraron que, si se correlaciona la violencia con la antigüedad laboral del profesional de enfermería evaluado $(r=0.227$; $\mathrm{p}=0.020)$, es decir a mayor antigüedad laboral, mayor percepción de ser víctima de violencia; en cambio los autores Purpora, et al ${ }^{31}$, presentan una correlación no estadísticamente significativa entre la cantidad de años trabajados y violencia horizontal $(\mathrm{r}=-0.11)$. Coincidente a esto, los resultados de Blackstock, et al ${ }^{34}$, demostraron que la antigüedad en la organización no se correlacionó con violencia horizontal.

Respecto al lugar de trabajo, el estudio coreano ${ }^{32}$, reportó que el personal de las UCI (Unidad de Cuidados Intensivos) médicas habían experimentado menos acoso laboral (1.47), en comparación con aquellos que están en Unidades de Cuidados Intensivos Especializados (1.75) y mixtas (1.69), con una correlación estadísticamente significativa. Lo anterior va en la misma línea respecto al estudio de Budin, et $\mathrm{al}^{5}$, quienes encontraron que las diferencias de acuerdo al lugar de trabajo fueron estadísticamente significativas, el $16.8 \%$ del personal de enfermería de la UCI presentaron moderado abuso verbal y el $91.9 \%$ de otros servicios clínicos manifestaron altos niveles de abuso verbal, esto quiere decir que el lugar o puesto de trabajo si influye en ser víctima de violencia horizontal. 
Un aspecto del lugar de trabajo que se debe destacar, corresponde a las horas a la semana que trabaja el profesional de enfermería, Oh, et al $^{29}$ demostraron que, quienes trabajaban entre 60-61 horas a la semana, tienen mayor riesgo de ser víctimas de abuso verbal.

Respecto a la satisfacción con el trabajo, los autores Purpora y Blegen ${ }^{30}$, indican que la satisfacción en el trabajo es inversamente proporcional con la violencia horizontal ( $\mathrm{r}=-0.462 ; \mathrm{p}<0.001)$, es decir, a mayor satisfacción laboral existía menor prevalencia de violencia horizontal. Hallazgo similar y con correlación estadísticamente significativa se reportó en el estudio de Armmer y Ball ${ }^{33}$.

Un aspecto de la satisfacción con el trabajo es la capacidad de brindar una atención de calidad, en donde los resultados de Purpora, et $\mathrm{al}^{31}$, demostraron una correlación inversamente proporcional entre la calidad de la atención y violencia horizontal ( $\mathrm{r}=-0.469 ; \mathrm{p}<0.001)$.

Esto demuestra que quienes son víctimas de actos de violencia presentan disminución de la calidad con la que brindan sus cuidados.

Por otro lado, Blackstock, et al ${ }^{34}$ reportaron que obstaculizar el trabajo hace que aumente la percepción de violencia horizontal, esto es estadísticamente significativo $(\mathrm{r}=0.64 ; \mathrm{p}<0.001)$. En efecto, Purpora, et $\mathrm{al}^{31}$ indicaron una correlación significativa, directamente proporcional entre violencia horizontal con errores y eventos adversos en la atención $(\mathrm{r}=0.442$; $\mathrm{p}<0.001)$, es decir, a mayor grado de violencia, aumenta la frecuencia de errores y eventos adversos generados en los cuidados hacia los usuarios.

Respecto a las relaciones entre pares, los autores Purpora y Blegen ${ }^{30}$ encontraron una correlación estadísticamente significativa e inversamente proporcional entre el puntaje de relaciones entre pares y violencia horizontal $(\mathrm{r}=-0.641 ; \mathrm{p}<0.001)$, por lo tanto, si es mejor la relación entre pares enfermeras, la percepción de violencia entre ellas disminuye.

En el estudio de Oh, et al ${ }^{29}$, el individualismo y experimentar emociones negativas por parte del personal de enfermería, víctimas de violencia, se correlacionaron significativamente con abuso verbal de la escala de violencia lateral. Es decir, aquellos profesionales de enfermería víctimas de maltrato laboral presentaban características individuales de baja autoestima, tendencia a la introversión, afecto negativo.

En relación a la idea de abandonar el trabajo, los resultados de Budin, et a $1^{5}$, plantean que los profesionales que están pensando en irse del lugar de trabajo se asocian a altos niveles de abuso verbal entre pares (pensar en irse antes de 12 meses: 18.6\% y/o irse antes de 3 años: 24.3\%). El estudio de Blackstock, et $\mathrm{al}^{34}$ va en la misma línea, ellos indican que a mayor violencia horizontal mayor intención del personal para abandonar su puesto de trabajo $(\mathrm{r}=0.32 ; \mathrm{p}<0.001)$.

Finalmente, respecto a las políticas de acoso laboral, los autores Yun, et al ${ }^{32}$, indicaron que el personal de enfermería de Unidades de Cuidados Intensivos que no cuentan con políticas o comités relacionados con el acoso en sus instituciones, habían sufrido más acoso laboral de pares que aquellas insertas en unidades con políticas o comités para evitar y tratar el acoso laboral (1.72 vs 1.38 ). Por otro lado, el hacer mal uso de los procesos de la organización por parte del profesional de enfermería, contribuía significativamente a aumentar la violencia horizontal, según lo indicado por Blackstock, et al ${ }^{34}$.

Con el objetivo de combinar resultados de estudios con homogeneidad clínica, se aplicaron dos metaanálisis con el método inverso de la varianza para resumir coeficientes de correlación. El primero fue para resumir la información de dos estudios equivalentes que correlacionaron edad y violencia horizontal, encontrándose homogeneidad estadística, por lo que la media ponderada se calculó con el modelo de efectos fijos, se obtuvo un valor de correlación global de 0.1545 y p $=0.0101$. No obstante, su significación estadística, presume que el valor del coeficiente es indicativo debido a que la correlación entre edad y violencia horizontal es bajo (Figura 2).

La Figura 3 presenta los resultados luego de combinar las correlaciones de tres estudios que utilizaban los coeficientes de Pearson, para experiencia laboral y violencia horizontal, no se observó una correlación estadísticamente significativa $(r=0.0697$; IC 95\%: $-0.1078-0.2428 ; p=0.4424)$. 


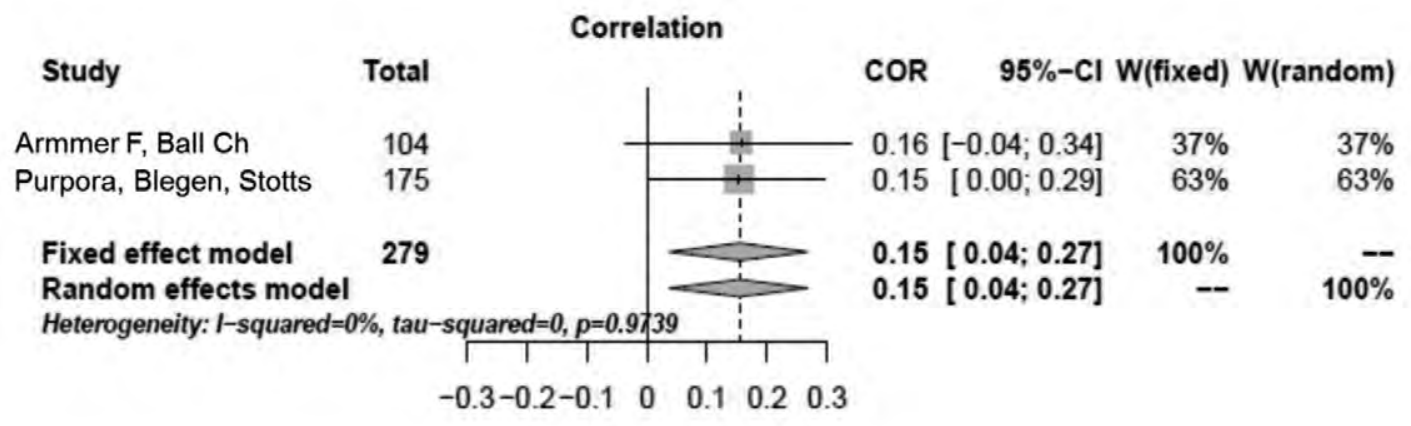

Figura 2. Forest plot de la correlación entre edad y violencia horizontal

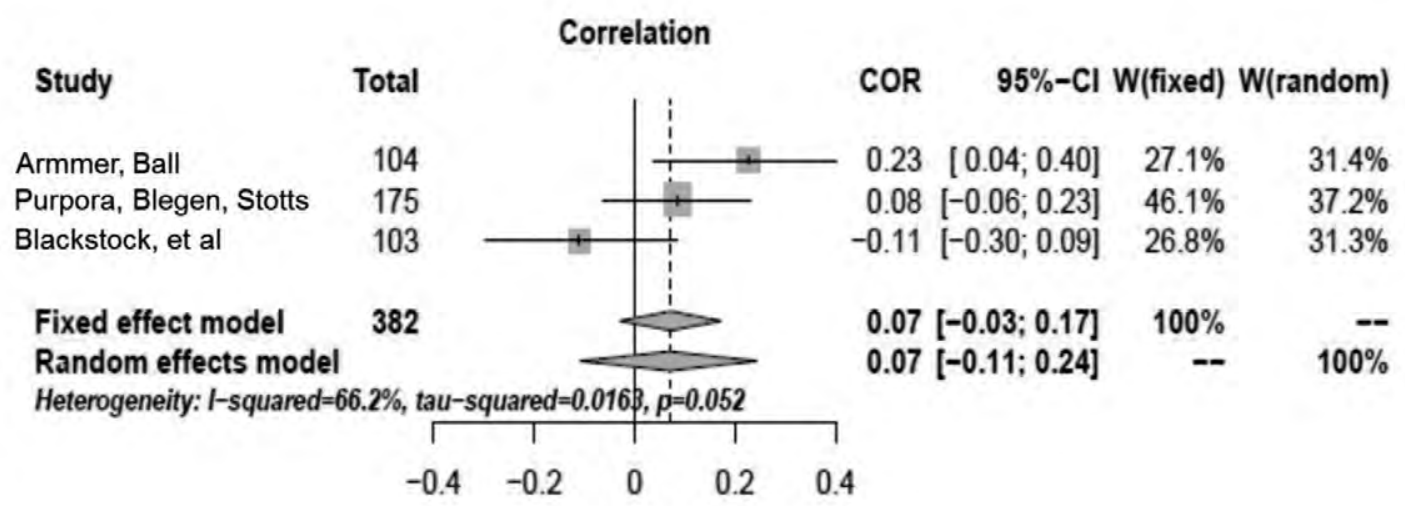

Figura 3. Forest plot de la correlación entre experiencia laboral y violencia horizontal

\section{Discusión}

Respecto a los resultados de esta revisión sistemática, primeramente cabe destacar la nula producción de artículos en español a diferencia de los encontrados en inglés, idioma de referencia en la comunidad científica. El análisis de la información recogida indica que la frecuencia de violencia o acoso horizontal oscila entre el $21.1 \%$ y $79.4 \%$, lo cual es concordante a la literatura revisada ${ }^{1,2,14}$. Entre los principales factores asociados a sufrir o ser víctima de violencia horizontal se encuentran: edad, lugar de trabajo, satisfacción con el trabajo, relaciones entre pares, plan para abandonar el trabajo y ausencia de políticas para denunciar el acoso laboral. Además, los estudios analizados, reportan que también existen otros factores de riesgo a considerar, y que se presentan en quienes son víctima de los actos de violencia tales como: tener una corta trayectoria profesional, trabajar en el área clínica en el turno de la noche ${ }^{35}$ y tener problemas de comunicación en el equipo de trabajo ${ }^{36}$. En variables como: género, estado civil y nivel educacional, la relación con violencia horizontal no es clara, por lo que sería interesante poder contar con más investigaciones que consideren estas variables y así poder medir su asociación.

Respecto a los dos metaanálisis realizados en esta revisión sistemática, el primero fue entre dos artículos que correlacionaron la edad y la violencia horizontal, tal como muestra la Figura 2, en el que se encontró una correlación positiva, es decir a mayor edad mayor percepción de violencia horizontal, pero esta correlación es baja (dos artículos), por lo tanto se debe interpretar con cautela.

El otro metaanálisis realizado (tres artículos) fue entre las variables experiencia laboral y violencia laboral, en este caso la correlación no fue estadísticamente significativa (Figura 2).

En relación al lugar de trabajo, los artículos estudiados son concordantes con la teoría, tal como lo demuestran Yun, et $\mathrm{al}^{32}$ y Budin, et $\mathrm{al}^{5}$. Dado que en el ambiente intrahospitalario hay una cantidad importante de unidades clínicas con un alto estándar de especialización médica y tecnológica, es altamente 
probable que se genere un ambiente de presión y estrés, que incide en el desgaste de las relaciones laborales.

En efecto, los resultados de este estudio plantean que a mayor edad se presenta una tendencia a obtener mayor puntaje de sufrir violencia. Sin embargo, esta relación puede incorporar factores de confusión, como la experiencia laboral, ya que los diseños de los estudios para esta RS son observacionales y con mediciones en tiempo transversal.

Una explicación al fenómeno de la presencia de violencia horizontal entre el personal de enfermería, es el que postulan los autores Croft y Cash ${ }^{9}$, indican que la falta de habilidades de gestión, por ejemplo; puede generar la internalización de una visión negativa de sí mismo (baja autoestima y la depreciación), que ocurre cuando se produce la adhesión al opresor e incapacidad de los oprimidos, por cuestionar las figuras que detentan el poder, encamina a que la agresividad acumulada se proyecte contra los miembros del propio grupo antes que contra el opresor. Este aspecto, a primera vista, es difícilmente reconocible y se hace evidente cuando se observa división y falta de cohesión en los grupos de enfermería ${ }^{37}$. No obstante, el mismo profesional mantiene el statu quo a través de la negación, minimización y ritualización, actitudes provocadas por las múltiples tareas que debe realizar el profesional, por consiguiente pasa por alto los efectos del abuso horizontal ${ }^{38}$.

Cabe destacar que de los siete artículos revisados, tres de ellos mencionan que la violencia horizontal tiene un modelo explicativo a través de la Teoría de la Opresión, esta teoría describe los contextos sociales que contribuyen a la agresión y violencia, el personal de enfermería, como grupo, son oprimidos pues han sido controlados por fuerzas externas a ellos mismos, que tuvieron mayor prestigio, poder o estatus y que han explotado al grupo menos poderoso ${ }^{37}$, lo cual es concordante a los expresado en el marco teórico de este estudio. Al igual que en otros grupos oprimidos, el personal de enfermería es a menudo silenciado al emitir sus propias contribuciones para la atención de los pacientes, y por lo tanto disminuye su propio sentido de valor, así como su capacidad para ofrecer una buena atención ${ }^{39}$.

\section{Conclusión}

La violencia horizontal, un concepto forjado por la teoría de la opresión, fue usada en este estudio, porque implica el comportamiento negativo entre pares que tienen la misma posición social en una institución, en este caso, los hospitales.

Dentro de las fortalezas de este estudio, se puede mencionar que con la información obtenida se pudieron realizar dos metaanálisis, pero como ya se mencionó anteriormente, los resultados de éstos deben ser tomados con cautela por el tipo de diseño de los estudios, ya que todos fueron de tipo transversal.

La violencia horizontal (ejercida por pares) es un grave problema que afecta a las enfermeras(os) que trabajan en hospitales. La prevalencia según este estudio, oscila entre el $21 \%$ y $79 \%$, lo cual demuestra que es un fenómeno de gran magnitud y que por ello requiere realizar investigaciones a futuro que aborden esta temática, sobre todo en idioma español, ya que se desconoce el alcance de este problema en países de habla hispana.

De acuerdo a lo revisado, los factores a tener en consideración y que se asocian a violencia lateral u horizontal son: edad, lugar de trabajo, satisfacción con el trabajo, relaciones entre pares, plan para abandonar el trabajo y políticas relativas al acoso laboral.

Pero se requiere más indagación, primero para comprender y reconocer esta problemática, después para el diseño de políticas de intervención que permitan mejorar la calidad de la atención y promover ambientes laborales saludables para enfermería.

\section{Responsabilidades éticas}

Protección de personas y animales. Los autores declaran que para esta investigación no se han realizado experimentos en seres humanos y animales, ya que se trata de un estudio realizado sólo con apoyo documental. 
Financiamiento. Ninguno

Conflicto de intereses. Los autores declaran no tener conflicto de intereses.

\section{Referencias}

1. Walrafen N, Brewer MK, Mulvenon C. Sadly caught up in the moment: an exploration of horizontal violence. Nurs Econ. 2012; 30(1): 6-12.

2. Reynolds G, Kelly S, Singh-Carlson S. Horizontal hostility and verbal violence between nurses in the perinatal arena of health care. Nurs Manag. (Harrow). 2014; 20(9): 24-30.

http://dx.doi.org/10.7748/nm2014.02.20.9.24.e1098

3. Christie W, Jones S. Lateral Violence in Nursing and the Theory of the Nurse as Wounded Healer. Online J Issues Nurs. 2014; 19(1): 5. https://doi.org/10.3912/OJIN.Vol19No01PPT01

4. Mckenna BG, Smith NA, Poole SJ, et al. Horizontal violence: experiences of Registered Nurses in their first year of practice. J Adv Nurs. 2003; 42(1): 90-6.

https://doi.org/10.1046/j.1365-2648.2003.02583.x

5. Budin W, Brewer C, Chao Y-Y, et al. Verbal abuse from nurse colleagues and work environment of early career registered nurses. J Nurs Scholarsh. 2013; 45(3): 308-16. https://doi.org/10.1111/jnu.12033

6. Vessey J, DeMarco R. DiFazio R. Chapter 6 Bullying, Harassment, and Horizontal Violence in the Nursing Workforce: The State of the Science. Annu Rev Nurs Res. 2010; 28(1): 133-57. https: / /doi.org/10.1891/0739-6686.28.133

7. Tovar-Bohórquez JO. Pedagogía del oprimido: escrito dirigido al opresor. Pensam cult. 2015; 18(1): 155-73. https://doi.org/10.5294/pecu.2015.18.1.6

8. Roberts SJ. Oppressed group behavior: implications for nursing. ANS Adv Nurs Sci. 1983; 5(4): 21-30.

9. Croft RK, Cash PA. Deconstructing contributing factors to bullying and lateral violence in nursing using a postcolonial feminist lens. Contemp Nurse. 2012; 42(2): 226-42. https://doi.org/10.5172/conu.2012.42.2.226

10. Lachman VD. Ethical Issues in the Disruptive Behaviors of Incivility, Bullying, and Horizontal/Lateral Violence. Medsurg Nurs. 2014; 23(1): 56-60.

11. Bortoluzzi G, Caporale L, Palese A. Does participative leadership reduce the onset of mobbing risk among nurse working teams? J Nurs Manag. 2014; 22(5): 643-52. https://doi.org/10.1111/jonm.12042

12. Sanner-Stiehr E, Ward-Smith P. Lateral violence in nursing: implications and strategies for nurse educators. J Prof Nurs. 2017; 33(2): 118-8. https:// doi.org/10.1016/j.profnurs.2016.08.007

13. Losa-Iglesias ME, Becerro de Bengoa-Vallejo R. Prevalence of bullying at work and its association with self-esteem scores in a Spanish nurse sample. Contemp Nurse 2012; 42(1): 2-10. https:// doi.org/10.5172/conu.2012.42.1.2

14. Vessey JA, Demarco RF, Gaffney DA, et al. Bullying of staff registered Nurses in the workplace: a Preliminary study for developing Personal and organizational strategies for the transformation of hostile to healthy workplace environments. J Prof Nurs. 2009; 25(5): 299-306. https://doi.org/10.1016/j.profnurs.2009.01.022

15. Chang HE, Cho S-H. Workplace Violence and Job Outcomes of Newly Licensed Nurses. Asian Nurs Res (Korean Soc Nurs Sci). 2016; 10(4): 271-6. https://doi.org/10.1016/j.anr.2016.09.001

16. Purpora Ch, Blegen MA. Horizontal Violence and the Quality and Safety of Patient Care: A Conceptual Model. Nurs Res and Pract. 2012; 5pp. http://doi.org/10.1155/2012/306948

17. Castranovo MA, Pullizzi A, Evans S. Nurse Bullying: A review and a proposed solution. Nursing Outlook. 2016; 64(3): 208-14. https://doi.org/10.1016/j.outlook.2015.11.008 
18. Camerino D, Estryn-Behar M, Conway PM, et al. Work-related factors and violence among nursing staff in the European NEXT study: A longitudinal cohort study. Int J Nurs Stud. 2008; 45(1): 35-50. https: //doi.org//10.1016/j.ijnurstu.2007.01.013

19. Embree JL, White AH. Concept Analysis: Nurse-to-Nurse Lateral Violence. Nurs Forum. 2010; 45(3): 166-73. https://doi.org/10.1111/j.1744-6198.2010.00185.x

20. Karatza C, Zyga S, Tziaferi S, et al. Workplace bullying and general health status among the nursing staff of Greek public hospitals. Ann Gen Psychiatry. 2016; 15:7. https://doi.org/10.1186/s12991-016-0097-z

21. Lin YH, Lui HE. The impact of workplace violence on nurses in South Taiwan. Int J Nurs Stud. 2005; 42(7): 773-8. https:doi.org/10.1016/j.ijnurstu.2004.11.010

22. Calvo-González G, Camacho-Bejarano R. La violencia de género: evolución, impacto y claves para su abordaje. Enferm. glob. 2014; 13(33): 424-39.

23. Vílchez-Barboza V, Paravic-Klijn T, Valenzuela-Suazo S. Contribución de Enfermería al abordaje de los trastornos psicosomáticos de la mujer trabajadora. Enferm. glob. 2013; 12(31): 254-64.

24. Scarone-Adarga M. Violencia laboral intramuros. Hostigamiento sexual y otras formas de violencia contra la mujer en las maquiladoras de Sonora y Baja California. Rev.Col.Sonora. 2014; 26(4): 129-54.

25. Dahlby MA, Herrick LM. Evaluating an Educational Intervention on Lateral Violence. J Contin Educ Nurs. 2014; 45(8): 344-50. https:doi.org//10.3928/00220124-20140724-15

26. Purpora C, Blegen MA, Stotts NA. Horizontal Violence Among Hospital Staff Nurses Related to Oppressed Self or Oppressed Group. J Prof Nurs 2012; 28(5): 306-14.

https:doi.org//10.1016/j.profnurs.2012.01.001

27. Von Elm E, Altman DG, Egger M, et al. Declaración de la Iniciativa STROBE (Strengthening the Reporting of Observational studies in Epidemiology): directrices para la comunicación de estudios observacionales. Gac Sanit. 2008; 22(2): 144-50.

28. Agency for Healthcare Rersearch and Quality (AHRQ). EPC Evidence-Based Reports. Rockville: U.S. Department of Health \& Human Services. (Consultado 19 abril 2016]. Disponible en: https: / / bit.ly/2yKuU6X

29. Oh H, Uhm DC, Yoon YJ. Factors affecting workplace bullying and lateral violence among clinical nurses in Korea: descriptive study. J Nurs Manag. 2016; 24(3): 327-35. https://doi.org/10.1111/jonm.12324

30. Purpora C, Blegen MA. Job satisfaction and horizontal violence in hospital staff registered nurses: the mediating role of peer relationships. J Clin Nurs. 2015; 24(15-16): 2286-94. https://doi.org/10.1111/jocn. 12818

31. Purpora C, Blegen MA, Stotts NA. Hospital staff registered nurses' perception of horizontal violence, peer relationships, and the quality and safety of patient care. Work. 2015; 51(1): 29-37. https://doi.org/10.3233/WOR-141892

32. Yun S, Kang J, Lee Y-O, et al. Work Environment and Workplace Bullying among Korean Intensive Care Unit Nurse. Asian Nurs Res (Korean Soc Nurs Sci). 2014; 8(3): 219-25. https://doi.org/10.1016/j.anr.2014.07.002

33. Armmer F, Ball C. Perceptions of horizontal violence in staff nurses and intent to leave. Work. 2015; 51(1): 91-7. https://doi.org/10.3233/WOR-152015

34. Blackstock S, Harlos K, Macleod M, et al. The impact of organisational factors on horizontal bullying and turnover intentions in the nursing workplace. J Nurs Manag. 2015; 23(8): 1106-14. https://doi.org/10.1111/jonm.12260

35. Pai HC, Lee S. Risk factors for workplace violence in clinical registered nurses in Taiwan. J Clin Nurs. 2011; 20(9-10):1405-12. https://doi.org/10.1111/j.1365-2702.2010.03650.x 
36. Molero-Jurado MM, Pérez-Fuentes MC, Gázquez-Linares JJ. Acoso laboral entre personal de enfermería. Enferm Univ. 2016; 13(2): 114-23. http://dx.doi.org/10.1016/j.reu.2016.03.001

37. Medina-Moya JL, Schubert-Backes VM, do Prado ML, et al. La enfermería como grupo oprimido: las voces de las protagonistas. Texto contexto enferm. 2010; 19(4): 609-17. http://dx.doi.org/10.1590/S0104-07072010000400002

38. Taylor B. Identifying and transforming dysfunctional nurse-nurse relationships through reflective practice and action research. Int J Nurs Pract. 2001; 7(6): 406-13.

https://doi.org/10.1046/j.1440-172X.2001.00323.x

39. Roberts SJ, Demarco R, Griffin M. The effect of oppressed group behaviours on the culture of the nursing workplace: a review of the evidence and interventions for change. J Nurs Manag. 2009, 17 (3): 288-93. https://doi.org/10.1111/j.1365-2834.2008.00959.x 